\title{
SHOULD THE PSYCHIATRIST BE HOSPITALIZED?
}

\author{
FREDERICK HAYES-ROTH \\ University of Michigan \\ RICHARD LONGABAUGH \\ Butler Hospital and Brown University \\ RALPH S. RYBACK \\ McLean Hospital and Harvard Medical School
}

W

E'VE been seeing the patient regularly for the past five years. For the sake of anonymity, we will call him Psi. His problem as initially presented to us was a chronic inability to use his full potential in his work. Particularly, he showed an inability to learn by his past mistakes. He would make the same errors time and time again. It appeared that he couldn't remember what caused him to behave the way he had on previous occasions, and couldn't see how the present situation was analogous to a prior one. He couldn't remember how he had previously responded to comparable situations. And he wasn't able to see what effects his behaviour had upon those he dealt with.

This lack of problem-solving performance was compensated for by ritualistic behaviours. He spent a great deal of time behaving in a manner which was intended to give an appearance of analysing his prior work performance. However, he never really learned anything from this ritual, and often would admit that the only reason he did this review was because his employer required it of him. However, at other times when the suggestion was made that he do this review differently, he would dogmatically assert that the way he presently did it was the right way, and should be rigorously adhered to because this was the way his employer wanted it to be done.

We watched him actually carry out his work. Our observations revealed first of all that he had considerable difficulty in isolating what was the problem to be solved. He was likely to state problems in overly general ways, such that they were not really capable of solutions. Secondly, even when he finally arrived at a statement of what the problem was, shortly thereafter he would of ten reverse himself and offer a contradictory statement of the problem. Often he would seem to forget what the initial problem was and give evidence of "flight of ideas".

In summary, it took him an overly long time to arrive at his evaluation of the problem.

One would hope that this protracted appraisal was perhaps due to the fact that he was thinking through his plan for attacking the problem, and that this would soon be evident. However, usually this was not the case, for his plans for dealing with the problem seemed to be somewhat independent of what the particular problem was. Typically, he chose one of two ways of dealing with the problem. Either he himself was going to try to fix it or not. If he was going to try to fix it himself, he would then spend considerable time determining where the work would be done. However, once that decision was made, it seemed to be unrelated to how he was going to fix it.

We were forced to wonder why so much focus on the where, and so little on the what. In most cases, the type of tools he planned to use depended upon how much the customer could pay. If the customer could afford his most elaborate tools then that is what he would use. If, however, customer resources were limited, he would give him the grade tools that the customer could afford. 
Once the decision as to what tools should be used was completed, he went to work. He would often work for a very long time on his product with considerable effort. However, this is where his chief problem arose: he never made a systematic study of whether the quality of his final product was related to the kinds of tools he used, the time he spent working on the problem solution, or the costs involved in the work.

As a consequence Psi continued his work in the same old ways, except that he did take to fads as they came along, use them for a while, and then discard them. As a result his work was extremely costly, but he had no idea as to what it was worth.

His employer became increasingly impatient with his work and first asked him to either document the value obtained for the cost, or if he couldn't, change his ways.

But Psi didn't respond to the suggestion, feeling that he himself best knew how to do his work, and that his employer should mind his own business.

When his employer (who really knew little of the details of Psi's work) asked Psi to furnish documentation of his work in terms of stating what it was that was wrong with what was to be fixed in the first place, what steps he took to fix it, and what effects his steps had on fixing it Psi would become quite irate. He would refuse to furnish this documentation, saying that it was really confidential information which his employer had no need to look at.

The result was that various customers decided that they would not pay their bills. Because several of the customers were not paying their bills, this raised the cost of the work that was done for the rest.

Moreover, several customers started looking to alternative solutions for getting things fixed. They started self-help groups, community services and the like. They increasingly shopped around for cheaper repair services as well.

Finally Psi's employer stepped in and told Psi that if he wanted to keep his job he was going to have to find ways of learning from his prior experience.

Now Psi is currently mulling over his problem. Some days of the week he maintains that there is no problem, that others are persecuting him, trying to control his behaviour; that he is already doing the best that he can do, which is a damn-sight better than any of his critics could do. He goes around talking to himself complaining that "they just don't understand". If they had his knowledge and insight, all would be revealed. However, he despairs of teaching them because they don't understand his language, the language which he uses when he is talking to himself.

At such times as these, his autism borders on the extreme.

On other days of the week, however, we see him in deep despair. He admits that there is indeed a problem, but that the problem is so complex he feels it may be impossible to solve. In fact, he suspects that it may, in principle, be insoluble.

On such days Psi may also candidly admit that he really has little knowledge to go on in his work, and that he plays hunches and uses his best intuitions for determining how he shall go about his tasks. He yearns to have a more solid basis on which to make decisions, but he is at a loss as to where such knowledge is to come from. He would very much like to know how the nature of his work relates to the quality of his product.

Despite the fact that he has all the secondary symptoms which arise from his primary problem of inability to learn from past experience, Psi is really a nice fellow. His heart is in the right place; he's a real humanitarian; he works extremely hard at his job, and he greatly cares about whether or not he is able to fix the products he is given to work on.

Prognostically, it is our clinical judgment that the goal of treatment for this 
patient is for him to learn and incorporate behavioural patterns which will involve getting the feedback whereby he can systematically learn from his prior work experience.

We have a treatment plan in mind that may well accomplish this treatment goal. When it is accomplished it is our belief that the secondary symptoms will disappear. At any rate it is worth a try.

The first step in this treatment plan is attempting to get through to Psi that there is a treatment for his problem, that the treatment process is long and tedious, but that if followed faithfully, in the long haul the problem is soluble.

Our recommendation is that if he does not commit himself to treatment and follow the treatment plan, the kindest thing we could do for him would be to hospitalize him.

For any of you who may still be in the dark as to who Psi is, he is the traditional Psychiatric Hospital.

Must we hospitalize the Psychiatric Hospital? Or is there an alternative method of treatment, which with long-term therapy will result in basic character changes in its personality?

We propose that a successful treatment can be planned and implemented which will bring about the long-term character changes necessary for more adaptive relationships of the psychiatric hospital to its environment.

The essence of the treatment plan is the development of an information system within the hospital. The function of this information system is to provide a vehicle whereby the hospital can systematically learn from its own experience.

In order to implement the treatment plan it is necessary that we have the tools, the resources, and that the patient have the motivation. We submit that all are currently available to us: the tools are the Problem-Oriented Medical Record and the Utilization Review Plan; the resources can be supplied by payment through Medicare-Medicaid reimbursement; the motivations lie in the practitioner's need for competency and mastery, and in the hospital systems' goal of survival.

\section{THE INFORMATION SYSTEM}

In order to learn by experience it is necessary that two events be reliably known and interrelated: The consequences of action and the actions themselves. As applied to the psychiatric hospital in its treatment of patients this means that: (1) the nature of the treatment provided for the patient be accurately characterized, and (2) the effects of the treatment upon the patient be accurately assessed.

While this seems like a simple enough task in the laboratory, as every one knows when such a dictum is applied to the "real world of psychiatric treatment" the task seems to become unmanageable and overwhelming. How can psychiatric treatment be adequately measured? And, even if a treatment could be sensibly assessed, how could its complex effects on a given patient ever be known, let alone adequately measured? Indeed, the task is difficult. But we have to start somewhere, and sometime. The "sometime" is now and the "somewhere" is here.

For those who deny that it is possible to assess psychiatric treatment and its effects, it should be pointed out that we presently act as if this is precisely what we do now. We hold planning conferences in order to determine the treatment a patient should receive. Such treatment plans are presumably based upon our best assessments of what the patient's problems are, and how they can best be treated. We hold treatment review conferences to assess what effects our treatments presumably have had upon the patient. Either our efforts have had some value and can be improved, or this is a pointless ritual. 
The job to be done is to take psychiatric treatment at its present level of sophistication, develop instruments to assess these treatments and treatment effects as they are presently perceived, and then over time and accumulative experience establish factual relationships between treatments and patient change.

In principle, the place where this information should be recorded and stored is in the medical record of the patient. In fact, the present medical record is not kept in such a way so that this is possible.

\section{THE CURRENT PSYCHIATRIC MEDIGAL RECORD}

The traditional psychiatric medical record suffers from numerous deficiencies.

It is not up to date because recording and dictation lie far behind events. The psychiatrist has neither the time nor the interest to spend on activities irrelevant to the patient's treatment. In current form the present record is virtually irrelevant to day-to-day patient care.

Its irrelevancy and its lack of timeliness are attributable to the information which is entered into the record, the information which is omitted from it and the way in which this information is organized. A reader either cannot find in the medical record the information he is looking for, or the information may be repeated several times in various places.

The repetition of information in the medical record is a problem in two ways. First, there is the unnecessary cost in entering the data numerous times. For example, in one hospital sampled, sex of patient is asked for on seven different occasions or forms, as is marital status, birthdate, religion, and legal status. Date admitted is recorded in nine separate documents and age is asked for in eight. Other information recorded in four or more places is social worker and physician assigned, ward admitted to, who the patient was accompanied by, patient's residence, length of patient's residence, location from which the patient was admitted, referring person, insurance coverage for patient (6 times!), and financial competency (i.e. ability to pay for the treatment, 5 times!).

The greater evil is that because one is not sure where in the record (let alone in the world!) the information may appear, it may be necessary to read the whole medical record from beginning to end in order to find out what it is you're looking for: Obviously, an unrealistic aspiration for a busy doctor or for any other busy member of the treatment team. (And we can assure you, all are very busy.)

An equally serious error in the current record is the error of omission. Because there is no standard body of information which is invariantly collected on a patient, one never knows whether the omission of information in the record is due to the fact that it never happened in the first place, or that it happened but was never recorded because someone (a) forgot, (b) didn't have time, or (c) didn't think the information to be sufficiently important. Random observations are of use only in a random treatment plan.

What can be better identified from the typical record is who has recorded the data: There may be a section of Nursing Data, Doctor's orders, and Doctor's Progress Notes, and several sections for the Social Worker. While the source of information should indeed be documented, it is much more important to organize the record by content than by content sources.

Only when the record is organized by specific topic areas will it be possible to easily locate what information is being sought. And then the information must be usable. A fact is insignificant in isolation; it must be related to another fact in order 
to determine useful relationships. Obviously, we have to develop a medical record for the psychiatric hospital which will enable us to relate our treatments to the patient's problems.

A radical change in medical record keeping is called for. Such a change has been proposed and developed by Lawrence Weed for the Medical Record of the General Hospital (1970). What remains to be done is for this method of record keeping to be adapted to the needs of the psychiatric hospital.

\section{THE PROBLEM-ORIENTED MEDICAL RECORD}

The philosophy underlying the Problem-Oriented Medical Record is that patients are treated for problems. Thus, a fundamental task of the record is to state what the patient's problems are. Secondly, treatments are for problems; thus, a treatment plan is developed for each of the patient's problems. Treatment is not in the abstract; it has a particular objective, the elimination or amelioration of the problem for which it is planned. Thus, the medical record should not only contain a list of problems to be treated, but also a treatment plan for each problem that is to be treated. From this it obviously follows that it should be possible to identify in the record which treatments are relevant to which problems. An easy solution is to number problems. In this manner everything else in the record can be related to a problem by indexing, using the problem number. For this reason Weed's ProblemOriented Medical Record uses a numbered problem list.

The problem-list identifies what the patient's condition was on a given date, prior to treatment. The treatment plan spells out what is going to be done for the patient in order to try to relieve his problem. The progress notes spell out what was done and the effects of what was done upon the patient. As new problems are identified, they are added to the list. As problems are solved, this too is recorded in the record at the time at which it is noted. Thus, at any point in time, by a quick look at the patient's record, one can find and determine: (1) what the patient's problems were and are, (2) what treatments were prescribed for each, (3) when any of the patient's problems were solved, and (4) what problems persist.

With such a record it is easily possible for members of a treatment review committee to know exactly what they're at, where they've begun, and where they must go.

As a method of building knoweldge, one can quickly cross-tabulate across patient records and discover what kinds of treatment effects are associated with what kinds of treatments. When this is done, we will find that a treatment which works for one patient may not work for another. In such a case, no easy relationship is obtained, and only the knowledge that relations are complex has materialized.

What does one do then?

Weed has provided for this possibility, because he follows an age-old, well-tested model; the scientific method. When one is "doing science", one tests for relationships between two or more events or variables within a given context. That is, one proposes that $\mathrm{X}$ will be related to $\mathrm{Y}$ under a certain set of conditions. Unless the set of conditions under which the relationship holds is specified, the generality of the relationship is unknown.

In relation to models based upon medical record findings, we realize that we must describe the specific context in which treatments are related to their effects. So, when we find that a given treatment results in changes in patient $\mathbf{P}$ but not in patient $\mathrm{O}$, we can then look to the differences with which the treatments were effected.

In order to look to the differences between patients, Weed's medical record 
includes a data base. This data base goes into the specifics concerning the patient and his prior history. Such a data base permits us to search for differences between the two patients which would enable us to hypothesise or explain why the treatment worked for patient $\mathrm{P}$ but not for patient $\mathrm{O}$. This kind of retrospective analysis will permit the refinement of hypotheses concerning relationships between treatments and their effects upon patients. Such hypotheses can be tested on future hospital admissions.

While more than just the category of patient may be necessary to specify the context in which treatment takes place, discussion of the full context will be reserved for the next section where the adoption of the problem-oriented medical record by the psychiatric hospital is discussed.

\section{A PROBLEM-ORIENTED MEDICAL RECORD FOR PSYCHIATRY}

Granted that the problem-oriented medical record is usable and useful for the general hospital, many psychologically-oriented mental health specialists will suggest that such a tool is not usable in psychiatry. The main reservations concerning adoption in this area focus on the seeming greater complexity in defining problems, treatments, and treatment effects. Let us discuss each in turn.

Psychiatric Problems

While the typical medical problem is seen as relatively well defined and clearly delineated, the typical psychiatric problem is seen as poorly defined, rather diffuse, and hard to separate from other psychiatric problems.

In general medicine the statement of the illness defines what has to be fixed. In psychiatry there is no easy, well-travelled road-map indicating the relationship between diagnostic illness and what problems have to be fixed. Thus, stating the patient's condition in terms of the problems he has which need to be solved, instead of in terms of his illness, will provide a partially new nomenclature for describing psychiatric conditions. Instead of a patient being vaguely defined as a "schizophrenic, simple reaction", he will be a person whose problems might include: (1) an inability to maintain sustained attention in problem-solving, (2) an inability to relate with effect appropriate to the interpersonal circumstances, (3) an inability to form close interpersonal relationships, and (4) an inability to sustain continued employment.

Each of these problems is reasonably clearly defined and delineated. And each provides a focus for intervention.

It will be noted that this problem list has two by-products: (1) it is slanted towards observable, molar events (e.g., he can hold a job or he can't), and (2) psychodynamics are not included as problems.

The push towards observables is a natural consequence of orienting the treatment towards the patient's problems. An undetectable problem is not a problem.

Does the absence of psychodynamics within the problem list mean that theory is being discarded? This will depend upon the orientation of the treatment agent. For a psychoanalytically oriented psychiatrist, psychodynamics will remain as a basic approach to problem treatment. Our own view is that the use of psychodynamics will become more clearly understood. A psychodynamic understanding of the patient will be the practitioner's theory as to why the patient has a given problem and how it can be resolved. For example, a patient's inability to maintain a close heterosexual relationship may be theorized to be the result of an unresolved oedipal complex. The treatment plans for this problem then might consist of individual psychotherapy directed at the patient's gaining insight into his problem, with these insights bringing about basic changes in personality structure. As a consequence of this treatment it is 
hypothesised that the patient's ability to form deep heterosexual relationships will be improved. The proof of this hypothesis will be provided when the sought-for relationship materializes.

In summary, problems can only be defined in terms of our present understanding of them. We must start at the beginning, but the beginning is where the patient feels it the most: in his inability to cope with the problems of everyday living. Treatments

At present, descriptions of treatments tend to be considered and stated in allpurpose terms, e.g. individual psychotherapy, milieu therapy, activities therapy, etc. As stated in the medical record, little is told about the goals or purposes of the treatment. This is because they have not been tied to specific problems. But there is no such thing as an all-purpose treatment. Rather, when treatments are proposed, they are theoretically proposed to solve a set of one or more problems.

When the goals of treatment are specified, they also provide the criteria by which the treatment can be evaluated. At the culmination of a treatment the problem list to be treated is reviewed, and it can then be determined whether the problems still exist or have been solved. Thus, our description of treatment plans must include the goals of each treatment.

We say "must" from a scientific point of view. But now we add "must" from a legal point of view, at elast as far as Medicare and Medicaid patients go. Specifying what are reimbursible treatment costs, the Hospital Manual of Health Insurance for the Aged states that there must be a plan of treatment which is recorded in the patient's medical record; the services the hospital provides the patient must be reasonably expected to improve the patient's condition, or be for the purpose of diagnostic study; the goal of therapy is improving the patient's condition where this is defined as the reduction or control of the patient's psychotic or neurotic symptoms which necessitated hospitalization and the improvement of the patient's level of functioning; that these treatment services be supervised and evaluated by a physician who must periodically evaluate the therapeutic programme in order to determine the extent to which treatment goals are being realized and whether changes in direction and emphasis are needed, and the treatment actually furnished the patient should be documented in the medical record in such a manner and with such frequency as to provide a full picture of the therapy administered as well as an assessment of the patient's reaction to it.

Thus, all the elements necessary for a systematic evaluation of the patient's treatment are required by law to be recorded in the medical record: the objectives of treatment, i.e. the problems of the patient which the treatment is reasonably expected to reduce; the treatment plan for the problem; the treatments actually furnished the patient (including their frequency); and, an assessment of the patient's reactions to the treatment.

We personally can think of nothing more reasonable to include. These elements are the same as those necessary for optimum clinical care, i.e., that the treatment agent know in as far as possible what problem he is attempting to treat; that he prescribe treatment plans for these specific problems, where the treatments selected are those reasonably expected to improve the patient's condition; that the actual treatment process be spelled out and tracked to see that it is implemented, and that the patient's response to the treatment be assessed, so that new treatment plans can be developed for the unresponsive patient.

One last point: it is important to emphasise again that Medicare-Medicaid determines whether or not the patient is receiving a reimbursable active treatment 
not only by what is done for the patient but just as importantly why it was done, i.e., what the purposes of the treatment were.

Treatment Effects

A third problem in adapting the problem-oriented medical record to the psychiatric hospital relates to the traditional difficulty of determining the patient's response to treatment. Improvement is less clearly determined in psychiatry than in general medical treatment.

But if the patient's condition is spelled out in terms of problems to be treated, the alleviation of the problems becomes the criterion of successful treatment. It is easier to assess whether the patient has more friends than he used to than to assess whether his schizophrenia is still manifestly present, has become latent, or has been removed.

In summary, the problem-oriented medical record can only help psychiatric treatment in that it will force us to specify more clearly what we are doing, why we are doing it, and what success our efforts are having. The result will be knowledge.

Once the data have been reliably and systematically recorded, we will have the data-base for answering the question of "what kind of patient problem is helped by what kind of treatment for what kinds of patients". Still remaining to be done is the building of a mechanism by which this question will be asked and answered. The requirements of the Medicare-Medicaid Programme call for just such a mechanism: The Hospital's Utilization Review Plan.

\section{Systematic Utilization Review}

In 1966 federal Social Security legislation required that hospitals implement Utilization Review Plans. The purpose of this U-R plan was to facilitate the appropriate utilization of institutional facilities and professional services. As designed, the plan called for a written review plan and utilization review programme for every partcipating hospital.

By and large, the experience with utilization review has been discouraging. At the very least, U-R has failed to accomplish its chief goal of ensuring efficient and quality care.

In the year of 1971 the Social Security Administration is increasing the pressure. In addition to the review of selected samples of individual patient records, a retrospective review of profiles and patterns of patient care is being required. These reviews are to be continuous and conducted systematically with rigour. They are to result in specific recommendations for changes in medical and administrative practices, and in the adoption of such recommendations. Hospitals which do not meet these requirements in their plan and implementation of utilization review in some states are subject to cancellation of licence.

In brief, the goal of these studies of group profiles is cost/effective treatment. Kinds of patients, patient problems or treatment categories are to be compared in order to determine what kind of patient with what kind of problem is effectively treated at what level of cost by what category or categories of treatment. Hospitals will be evaluated not only by the fact that such studies are going on, but that they result in concrete recommendations for changes in practice and for the implementation of such changes.

Pressure for cost/effective treatments is going to continue and increase from the federal and state governments. Moreover, private medical insurance companies will be increasingly inclined to follow the lead of government and ask that hospitals do the same for them that they are required to do for public health patients. 


\section{THE BENEFITS FROM USE OF THE PROBLEM-ORIENTED MEDIGAL REGORD} AND UTILIZATION REVIEW

There are immediate and direct benefits from use of the problem-oriented medical record to treatment team, patient, and hospital administration. The treatment team will benefit in that: (1) their use of the problem-oriented medical record will enable them to more clearly identify what is to be treatment and how it is to be treated, (2) they will have a better capacity to systematically determine what treatments were actually carried out, and what their effects were, (3) they will save time in that they will be able to consult the record and immediately find out what is going on with the patient and therefore will need fewer direct communications with one another trying to determine what is going on.

The patient will immediately and directly benefit in that his treatment plans will be better formulated and will be more responsive to changes in his conditions and problems. He will have more contact with staff who will have more time to devote to his direct treatment, and who will be better co-ordinated and informed in their knowledge and implementation of his treatment plan.

The Hospital Administration will immediately and directly benefit from: (1) the reduced cost of a shorter, comprehensive medical record, (2) reduced costs in better documentation of treatment for Medicare-Medicaid and private insurance company claims, (3) meeting the requirements of Public Health Licensure and MedicareMedicaid Certification, and (4) improved ability to meet the new mental health laws requiring documentation of periodic clinical reviews.

Indirectly and eventually there will be benefits from an effective utilization review plan. The clinical staff of a given hospital will collectively benefit from knowledge as to what treatments are more effective for what category of problems, and hence will gain greater satisfaction from their more successful problem solving techniques. The mental health community will benefit from knowledge as to what kind of problems benefit most from the kind of treatments a particular psychiatric facility provides. The hospital system will benefit in that more effective utilization of services will lower the costs of treatment. The patient will benefit from more effective treatment.

\section{The Costs of an Alternative Forgone}

What are the costs to the traditional psychiatric hospital of not implementing such an information system as is available from the problem-oriented medical record and utilization review plan? Research is beginning to point to an answer. We cite two studies: Those carried out by May (1968) and Pasamanick (1967) on treatment of schizophrenics, the most frequent category of patient occupying psychiatric hospital beds. May found the following. In terms of effective treatment:

\section{$\mathrm{D}+\mathrm{M}=\mathrm{D}+\mathrm{P}+\mathrm{M}>\mathrm{P}+\mathrm{M}>\mathrm{M}$.}

That is, drugs and hospital milieu (M) was as effective in treatment of schizophrenics as was the combination of drugs (D) and psychotherapy (P) and hospital milieu (M). Both combinations were more effective than the combination of psychotherapy (P) and hospital milieu (M) which in turn was better than hospital milieu (M) alone. In terms of costs May found that:

$\mathrm{D}+\mathrm{M} \leq \mathrm{D}+\mathrm{P}+\mathrm{M} \leq \mathrm{M}=\mathrm{P}+\mathrm{M}$.

The combination of drugs and hospital milieu was cheaper than the combination of drugs, psychotherapy and hospital milieu, which in turn was cheaper than the combination of psychotherapy and hospital milieu, or than hospital milieu alone. Thus, the most cost/effective treatment was drugs plus hospital milieu. 
Pasamanick compared hospital treatment with treatment at home with and without use of drugs for schizophrenics. Concerning effectiveness, he found:

$\mathrm{D}>\mathrm{D}+\mathrm{M}$.

The combination of drugs and home care was more effective than the combination of drugs and hospital milieu.

Concerning costs Pasamanick found:

$\mathrm{D}+\mathrm{M}>\mathrm{D}$.

Drugs and home care was cheaper than drugs and hospital milieu care. Thus, drugs and home milieu care was more cost/effective than drugs and hospital milieu.

Putting the studies of May and Pasamanick together, the implication that could be drawn concerning the treatment of schizophrenia is that the average schizophrenic person is most cost/effectively treated by the combination of drugs and home milieu.

Proponents of traditional psychiatric hospitalization will (and have) raise(d) objections to each of these studies and their conclusions. However, the burden of proof increasingly rests upon such proponents of traditional hospitalization. Unless these proponents document their assertions with utilization review studies and other research, the verdict will go by default. In such an event it will be too late to hospitalize the traditional psychiatric hospital. It will already be extinct.

\section{BIBLIOGRAPHY}

May, Philip R. A.: Treatment of Schizophrenia: A comparative study of five treatment methods. Science House, New York, 1968.

May, Philip R. A.: "Cost Efficiency of Treatments for the Schizophrenic Patient", Amer. J. of Psychiatry, 1971, $127(10): 118-121$.

Pasamanick, Benjamin, Frank R. Scarpitti, and Simon Dinitz: Schizophrenics in the Community. AppletonCentury-Crofts, New York, 1967.

Weed, Lawrence L.: Medical Records, Medical Education, and Patient Care. The Press of Case Western Reserve University, Ohio, 1970. 